\title{
Diaphragmatic involvement in hemiplegia and hemiparesis
}

\author{
AMOS D. KORCZYN, GEORGE HERMANN, AND RUTH DON \\ From the Neurology Department, Beilinson Hospital, Petah Tikva, and the Department of Physiology \\ and Pharmacology, Tel-Aviv University Medical School; the Roentgenology Department, Hadassah \\ University Hospital, Jerusalem; and the Department of Research and Statistics, \\ Kupat Holim Center, Tel-Aviv, Israel
}

Unilateral underactivity of the respiratory muscles in hemiplegia, although already described by Hughlings Jackson (1895), is still only rarely known to clinicians. We have shown (Korczyn, Leibowitz, and Bruderman, 1969) that on the paretic side of hemiplegics, movements of the diaphragm are of smaller amplitude, both spontaneously and in hyperventilation. Smith (1964), reviewing chest radiographs of 21 hemiplegics, concluded that 'there is suggestive evidence that the diaphragm may be involved in some cases of hemiplegia'. We have now tried to make a statistical analysis of the position of the diaphragm in a larger series of patients.

\section{MATERIALS AND METHODS}

All available chest radiographs of patients with hemiparesis or hemiplegia of vascular origin hospitalized in the Hadassah University Hospital in Jerusalem during the period from 1953 to 1967 were reviewed. In many cases there was more than one radiograph of each chest. Most of them had been made at deep inspiration. All the radiographs were reviewed by one of us (G.H.), who did not know which was the clinical side affected. It was decided that a difference of at least one intercostal space between the levels of the peaks of the domes of the hemidiaphragms would be considered significant.

Chest radiographs of a group of patients with no known neurological deficit, who had been hospitalized for fracture of the head of the femur were taken as a control group. Chest radiographs were made in the supine position, usually in full inspiration, in both groups. Patients with clinical or radiographic evidence of severe cardiopulmonary or abdominal disease were eliminated. After this elimination, 190 test patients and 100 controls were available.

Statistical evaluation was made by using $\chi^{2}$ tests.

\section{RESULTS}

CONTROL GROUP In the 100 control radiographs, both hemidiaphragms were at the same level in 61 . In 23 , the right hemidiaphragm was significantly higher; in 16 there was a relative elevation of the left hemidiaphragm. The difference between elevation of the right and left hemidiaphragm in this control group is not statistically significant $\left(P>0.1, \chi^{2}=1.26\right)$. However, there is a tendency for the right hemidiaphragm to be elevated more frequently than the left.

TEST GROUP In the radiographs of the 190 test patients, both hemidiaphragms were at the same level in $86(45 \%)$; there was elevation on the weak side in $66(35 \%)$ and on the normal side in $38(20 \%)$. There was thus significant elevation of one hemidiaphragm (on the normal or paretic side) in 104 patients $(55 \%)$. Statistically, the difference between this group and the control group was significant $(P<0.02, G=5.9109)$. In checking the test group itself, the difference between elevation on the weak side ( 66 patients, $35 \%$ ) and on the normal side (38 patients, $20 \%$ ), was also found to be statistically significant $\left(P<0.01 ; \chi^{2}=7.54\right)$.

CORRELATION BETWEEN SEVERITY OF LESION AND ELEVATION OF DIAPHRAGM All patients were classified as 'hemiplegic' or 'hemiparetic' and according to the elevation of a hemidiaphragm on the normal or weak side (see Table I).

In $38 \%$ of hemiplegics there was an elevation on the weak wide, as compared with only $32 \%$ of the

\section{TABLE I}

CORRELATION BETWEEN SEVERITY OF NEUROLOGICAL DEFICIT AND DIAPHRAGMATIC INVOLVEMENT

\begin{tabular}{llll}
\hline & Hemiplegia & Hemiparesis & Total \\
\cline { 2 - 4 } Elevation on weak side & $29(38 \%)$ & $37(32 \%)$ & $66(35 \%)$ \\
Elevation on normal side & 15 & 23 & 38 \\
No elevation & 32 & 54 & 86 \\
\hline Total & 76 & 114 & 190 \\
\hline
\end{tabular}


hemiparetics. This difference was not statistically significant $(P>0.30 ; \mathrm{G}=1.024)$. When the hemiparetic group was considered alone, the elevation of the hemidiaphragm was found to be more frequent on the weak side than on the normal. This was of borderline significance $(P>0.05)$. For the hemiplegic subgroup, the difference between elevation on the normal or weak side was statistically significant $\left(P<0.05 ; \chi^{2}=4.45\right)$.

EFFECT OF THE SIDE OF THE LESION ON THE DIAPHRAGM Patients were classified according to the side of the neurological deficit as compared with the side of hemidiaphragmatic elevation (see Table II).

TABLE II

EFFECT OF SIDE OF LESION ON DIAPHRAGMATIC INVOLVEMENT

\begin{tabular}{|c|c|c|c|c|}
\hline & & \multicolumn{2}{|c|}{ Side of elevation of hemidiaphragm } & \multirow{2}{*}{ No elevation } \\
\hline & & Right & Left & \\
\hline \multirow{2}{*}{$\begin{array}{l}\text { Weak } \\
\text { Side }\end{array}$} & Right & 31 & 24 & 44 \\
\hline & Left & 14 & 35 & 42 \\
\hline \multicolumn{2}{|c|}{ Control } & 23 & 16 & 61 \\
\hline
\end{tabular}

In both subgroups (right- and left-sided weakness), the hemidiaphragm on the paretic side tends to be elevated. However, on the right side this failed to reach statistical significance when compared with the control group $(P>0.05)$. In left hemiplegics and hemiparetics, the left hemidiaphragm was elevated on the left in more patients than in the control group. Comparing right-sided elevation, left-sided elevation, and no unilateral elevation, this group differs significantly $(P<0.01)$ from the control group.

UNILATERAL PNEUMONIA If one side of the diaphragm is hypoactive, it may be anticipated that unilateral pneumonia will be more frequent on this side. In the control group, no unilateral pneumonia was evident. Among our patients, unilateral pneumonia was radiographically evident in 28 patients (see Table III). Pneumonia was thus more frequent on the weak side (17 cases) than on the normal side (11 cases), and was also more frequent on the right than on the left. However, this fails to reach statistical significance.

The patients with unilateral pneumonia have sometimes shown elevation of the hemidiaphragm on the side of infection. All of these 28 patients were not included in our test group of 190 patients (Test Group above), as explained under Methods,
TABLE III

UNILATERAL PNEUMONIA IN HEMIPLEGICS

\begin{tabular}{lccc}
\hline & Right & Left & Total \\
\cline { 2 - 4 } Pneumonia on weak side & 11 & 6 & 17 \\
Pneumonia on normal side & 6 & 5 & 11 \\
\hline Total & 17 & 11 & 28 \\
\hline
\end{tabular}

but if they had been included, no difference in the statistical significance of our results would have been found from those presented above.

\section{DISCUSSION}

From the results presented above it can be concluded that in hemiplegia unilateral elevation of the corresponding hemidiaphragm exists more frequently than could be ascribed to chance alone. The left hemidiaphragm is involved in left hemiplegia much more frequently than the right hemidiaphragm in right hemiplegia. This may be due to asymmetry of representation: whereas the left hemidiaphragm is represented mainly in the right motor cortex, the right hemidiaphragm derives corticospinal efferents from both hemispheres of the brain. A more plausible explanation, however, is that in right hemiplegia upward movement of the corresponding hemidiaphragm is limited by the ligaments attaching it to the liver, whereas this does not happen on the left side. Moreover, the relative elevation of the hemidiaphragm on the weak side during inspiration is, at least partly, due to the pressure applied to it by the visceral content. Again, the right hemidiaphragm is protected by the liver from this pressure.

A third alternative explanation is that patients with right-sided weakness had, on the average, a lesser neurological impairment in general than patients with left-sided weakness. This assumption is based on the observation that in most series left hemisphere disease is more common than right hemisphere disease, possibly due to the fact that smaller lesions in the 'dominant' hemisphere come to attention. However, in our series the numbers for these two groups are almost equal (99 right-, 91 left-sided weakness), so that this explanation does not seem to have a decisive role.

Physiological experiments may help in further evaluating the importance of the motor cortex in respiratory regulation. Though the cerebral cortex of cats is inhibitory to respiration (Leibowitz, Korczyn, and Bergmann, 1965), electrical stimulation of the motor cortex was not found to cause hyperventilation (Ledsome and Mortimer, 1966). 
Whether or not our findings have clinical importance awaits further studies. It may be that the ventilation-perfusion ratio is disturbed by unequal aeration of both lungs. However, this seems rarely, if at all, of prime significance.

The relative incidence of lobar pneumonia on the right and left sides are given as $58 \%$ and $42 \%$ respectively (figures compiled from Jürgensen, 1875). Aspiration pneumonia is also more frequent on the right than on the left side, although we could not find exact figures. It is difficult, therefore, to have a proper control group for our group of unilateral pneumonia. If such a group were available, it is possible that left-sided pneumonia would be more common in left hemiplegics than could be ascribed to chance. In our small series of unilateral pneumonia patients the expectation that on the hypoventilated side pneumonia would be more common was not validated. This paucity of cases suggests that the importance of the unilateral hypoventilation in producing pneumonia is only marginal.

\section{SUMMARY}

In patients with hemiplegia or hemiparesis, asymmetry of the hemidiaphragms is more common than in a control group. This is due to elevation of the left hemidiaphragm in left-sided weakness. Unilateral pneumonia seems to occur more frequently on the left side in left hemiplegics and on the right in right-sided weakness.

Thanks are due to N. Strulovici, M.P.H., for his kind help.

\section{REFERENCES}

Heffron, R. (1939). Pneumonia, with Special Reference to Pneumococcus Lobar Pneumonia, p. 107. The Commonwealth Fund: New York.

Jackson, H. J. (1895). Superior and subordinate centres of the lowest level. Lancet, 1, 476-478.

Jürgensen, T. (1875). Croupous and catarrhal pneumonia. Cyclopedia of the Practice of Medicine, (5:3). Edited by H. V. Ziemssen. William Wood: New York. Quoted by Heffron (1939).

Korczyn, A. D., Leibowitz, U., and Bruderman, I. (1969). Involvement of the diaphragm in hemiplegia. Neurology (Minneap.), 19, 97-100.

Ledsome, J. R., and Mortimer, L. (1966). The respiratory effects of stimulating the motor cortex of the cat. J. Physiol. (Lond.), $185,57-58 \mathrm{P}$.

Leibowitz, U., Korczyn, A. D., and Bergmann, F. (1965). Effect of brain transections on the respiratory response to nerve stimulation. J. neurol. Sci., 2, 241-252.

Smith, M. (1964). The effect of hemiplegia on the diaphragm. Am. Rev. resp. Dis., 89, 450-452. 